\title{
Enforcing Architecture Constraints
}

\author{
Robert Balzer \\ Information Sciences Institute \\ balzer@isi.edu
}

\begin{abstract}
We have built an infrastructure which reifies the architectural connections between modules into a data structure that constantly reflects the dynamic structural architecture of a software system. It similarly makes available all communications passing through those connectors.
\end{abstract}

Since these connections and the communications passing through them constitute the entire behavior of an architecture, our infrastructure provides the basis for a full range of architecture-level monitoring, testing, debugging, and enforcement tools.

This paper concentrates on the latter by presenting a language for stating architectural constraints which can be checked against the data structure that dynamically reflects the software system's structural architecture.

\section{Introduction}

We have argued for some time that Instrumented Connectors were key to moving software architectures beyond documentation and static configuration. Even if the software architecture specification described a system's dynamic architectural aspects (its connection and communication behavior), without the ability to bind that specification to the actual behavior of the system, that description couldn't be used by tools as a formal specification against which to compare and contrast the system's actual behavior.

Instrumented Connectors provide that critical link to the runtime architectural behavior of a system. By providing access to the connect and disconnect events that establish and destroy connections between modules, and to the communicate events that pass through those connections, they allow tools to monitor all of the system's architectural behavior. We have constructed and previously reported on two architecture tools which utilize this behavior access. The first tool, the Architecture Animator, graphically animates the system's architecture to help people visualize its behavior. The second, the Architecture Driver, records a system's architecture behavior, creates a test harness for exercising some portion of that system, and tests that portion by using the recorded behavior as a scenario of inputs to be given to and outputs to be received from the live portion. It detects deviations (as defined by a user supplied "significant-difference" routine) from the recorded scenario and invokes a user supplied "off-scenario" routine when such a deviation occurs.

\section{Instrumented Connectors}

These architectural tools were limited by the unavailability of Instrumented Connectors. As a temporary workaround, we created a network socket based "mirror" component that acted as a relay between two communicating system modules by replicating all connects, disconnects, or inputs received from one as connects, disconnects, or outputs to the other. While replicating these architectural events, these "mirror" components also passed them along to our architecture infrastructure for recording or use in one of the architecture tools. By manually configuring them into a software architecture as intermediaries between each pair of communicating modules we were able to simulate the existence of instrumented network socket connectors.

We now have a real implementation of Instrumented Connectors, designed to span all communication based intermodule connectors (i.e. shared memory is excluded), provided at the operating system level. Currently, this implementation handles pipes, network sockets, and files, and work is under way to include Remote Procedure Calls (RPC).

This operating system level implementation allows us to support a wide variety of types of architectural connectors for all applications running on that operating system, and does so without having to modify those applications in any way. Unfortunately, it also makes the implementation platform specific and we must reimplement it for other platforms. The current implementation works on Sun UNIX and we plan to implement it on Microsoft Windows as well.

\section{Enforcing Architectural Constraints}

The existence of these Instrumented Connectors and our infrastructure which reifies the architectural connections between modules into a data structure that constantly reflects the dynamic structural architecture of a software system makes it very easy to construct a tool which enforces architectural constraints. In fact, because of the unique structure of our Relational Abstraction [Goldman92] specification language, this "tool" consists of nothing more than installing the architectural constraints to be enforced and a call back, invoked whenever one of these constraints is violated, to the infrastructure to prevent the current architectural event from completing.

\section{Why Relational Abstraction Makes This So Straightforward}

In Relational Abstraction, the state of an application is simply a set of facts (assertions). This state is changed by adding and/or removing facts from the state (changing a fact occurs by deleting the old fact and adding the new "changed" fact). Constraints limit the set of states the application can enter. That 
is, an application can not transition to any state that violates a constraint. Should it try to do so, an exception is raised and a "repair" associated with the constraint being violated is executed which can either augment the transition so that the constraint is no longer violated in the resulting state, or it can abort the transition (which causes the state to be rolled back to the wellformed state that existed before the transition).

Thus, by hooking our Instrumented Connector infrastructure to a Relational Abstraction application which expresses each architecture action as a transition that removes and adds appropriate facts, the state of this application will reflect the dynamic state of the system's architecture. This Relational Abstraction application has already been written and integrated with our temporary workaround network socket "mirrors" to support our two existing architectural tools which utilize this dynamic Relational Abstraction state.

By simply importing architectural constraints they will automatically be enforced as architecture behavior transitions this application from state to state.

\section{Example Architectural Constraints}

Notation: We will use single capital letters to denote literals and single lower case letters to denote variables.

\section{Require (connect A B)}

Module A must always (i.e. statically) be connected to Module B. If a particular type of connection is required (e.g. network socket) then that type of connection can be explicitly specified (e.g. (network-socket-connect A B)). An inference rule defines "connect" as the logical union of each of these specific types of connections.

\section{Require (or (connect A B) (connect A C))}

Module A must always be connected to Module B or Module $\mathrm{C}$ (or to both of them). The Exclusive-Or operator can be used to prevent simultaneous connections to both of these modules. ${ }^{1}$

\section{Require (implies (connect A C) (connect A B))}

Module A can only be connected to Module $\mathrm{C}$ while Module A is connected to Module B (logically, A connected to C implies that $\mathrm{A}$ is connected to $\mathrm{B}$ ).

\section{Require (x y) (implies (connect x y) (allowed-connect $x$ y))} Don't allow any connection between modules " $x$ " and " $y$ " to occur unless a connection between them is explicitly allowed (via the allow-connect assertion). This constraint ensures that only those connections specified in the architecture description can be formed during the execution of the system. The installation of the architecture specification into this enforcement tool must translate the explicitly allowed connections in that architecture into allow-connect assertions so that this constraint can prohibit all others.

\section{Prohibit (x y z w)}

(and (connect $x y$ ) (architecture-level $x \mathrm{z}$ ) (architecture-level y w) (> (absolute (- z w)) 1))

Don't allow connections in a layered architecture between non-adjacent layers (literally, a connection between modules " $x$ " and " $y$ " in architecture levels " $z$ " and " $w$ " respectively cannot occur if the absolute difference is between " $z$ " and " $w$ " is more than one). This constraint would use assertions of architecture-level from the architecture specification which assigned modules to the various levels of that layered architecture. Alternatively, this constraint could be enforced by the previous constraint (prohibiting connections not explicitly allowed) by translating the architecture layer specifications into allow-connect assertions for modules in adjacent layers and ensuring that these assertions are not created for any modules in non-adjacent layers. The advantage of stating this architecture-level constraint explicitly is that it would apply to any dynamically

generated modules as well as those statically configured into the architecture.

\section{Prohibit (x) (pipe-connect-chain x x) where (y z) (implies (pipe-connect y z)
and $(\mathbf{y} z \mathbf{z})$ (implies (and (pipe-connect-chain y z) (pipe-connect-chain $\mathrm{z}$ w)) \\ (pipe-connect-chain y w))}

Don't allow any loops in a chain of modules connected by pipes (literally, don't allow a pipe-connect-chain to be connected to itself). This constraint uses two inference rules. The first maps pipe-connects into pipe-connect-chains. The second forms the transitive closure of these chains by inferring that " $y$ " is pipe chain connected to " $w$ " if it pipe chain connected to some module " $\mathrm{z}$ " and that module is pipe chain connected to "w."

\section{Conclusion}

Our point is that once architectural behavior has been reified into a data structure that constantly reflects the dynamic architectural state, existing constraint formalisms provide an adequate basis for stating and enforcing many types of architectural constraints.

We have created a UNIX based infrastructure which does the former and are working on a "Installer" tool which translates an architecture specification into a set of architectural constraints in our Relational Abstraction formalism to be enforced by its existing consistency management facilities. Our intent is to use ACME as the architecture specification language accepted by this tool so that our enforcement service will be available for any ADL translatable into ACME.

We have illustrated the simplicity and power that can be achieved with existing constraint formalisms in enforcing architectural constraints on the dynamic connection topology of an architecture. Although this formalism currently has no temporal operators, the architecture's dynamic state can be used to conditionalize constraints (through the implies operator) so that they are only applicable in those particular states. Moreover they can be used to constrain dynamic connections between modules and the dynamic creation and destruction of modules.

We believe that the communications passing through these connections can be similarly reified and constrained by "protocol" specifications. However, the reification must be enriched beyond the current state of the architecture to include the sequence of communication events that have occurred in the connection as the constraints will range over these sequences. 


\section{References}

Goldman, Neil and Narayanaswamy, K. (1992) "Software Evolution through Iterative Prototyping" Proceedings of the $14^{\text {th }}$ International Conference on Software Engineering, Melbourne, Australia, May 1992.

\footnotetext{
1 All constraint systems must define the points at which the declared constraints are enforced. In particular, some mechanism (like transactions or atomic updates) must be provided to prevent them from being applied during a series of changes that moves the system from one well formed state to another. Without such a compound update mechanism there is no way to switch an exclusive-or connection. Because we intend to apply this constraint enforcement mechanism to unmodified operational systems, the specification of the compound update boundary must exist outside the operational system. Our current proposal is to require architectural wellformedness only when communication occurs between modules (i.e. not during a sequence of connects and disconnects).
} 